\title{
Effect of CEACAM-1 knockdown in human colorectal cancer cells
}

\author{
ZHONG-MIN HAN, HE-MEI HUANG and YONG-WU SUN \\ Department of Medical Technology, Zhengzhou Railway Vocational and Technical College, \\ Zhengzhou, Henan 450052, P.R. China
}

Received May 5, 2017; Accepted January 23, 2018

DOI: $10.3892 / \mathrm{ol} .2018 .8835$

\begin{abstract}
Carcinoembryonic antigen-related cell adhesion molecule 1 (CEACAM-1) is the major antigen of the CD66 cluster of granulocyte differentiation antigens. The present study aimed to assess the biological function of CEACAM-1 on the growth of human colorectal cancer (CRC) cells in vitro. Treatment of cultured CRC HCT-8 cells with CEACAM-1-specific siRNA successfully downregulated CEACAM-1 expression by $61 \%$ compared with control cells. The effects of CEACAM-1 downregulation on HCT-8 cell proliferation and apoptosis were then assessed via Cell Counting kit- 8 assay and flow cytometry, respectively. The results demonstrated that siRNA-induced CEACAM-1 downregulation significantly inhibited proliferation and increased apoptosis, but had no significant effect on cell cycle progression in HCT- 8 cells. Together, these results suggest that CEACAM-1 activity is critical to CRC growth, and thus, CEACAM-1 may be a promising therapeutic target for the treatment of CRC.
\end{abstract}

\section{Introduction}

Malignant tumor growth is a common and serious disease condition that may affect any number of different organs and tissues throughout the body, and incur varied and complex symptoms that are often life threatening. Colorectal cancer (CRC) is one of the most common gastrointestinal malignancies, and localizes to the rectum or the junction of the rectum and the sigmoid colon. In fact, CRC is the fourth most common type of cancer internationally, following gastric, esophageal, and lung cancer $(1,2)$.

Malignant tumors are often characterized by both tissue architecture disruption, and differentiation derangement. Cell adhesion dysregulation contributes to tumor invasion and metastasis (3), such that the abnormal expression of

Correspondence to: Dr Zhong-Min Han, Department of Medical Technology, Zhengzhou Railway Vocational and Technical College, 9 Qiancheng Road, Zhengzhou, Henan 450052, P.R. China

E-mail: hanzhongmin1@163.com

Key words: colon cancer, carcinoembryonic antigen-related cell adhesion molecule 1, HCT- 8 cell line, small interfering RNA various cell adhesion molecules induces a loss of cell-cell binding, thereby promoting tumor differentiation and malignant invasion (4). Carcinoembryonic antigen-related cell adhesion molecules (CEACAMs) are members of the glycosylphosphatidylinositol (GPI)-linked immunoglobulin (Ig) superfamily (5). CEACAM-1 is a CEACAM subtype that is also known as biliary glycoprotein I or CD66a $(6,7)$. Previous studies have demonstrated that CEACAM-1 expression is reduced in several tumor types, such as melanoma, lung, colon and ovarian cancer, compared with the corresponding normal tissues (8-14). This suggests that CEACAM-1 may function to inhibit carcinogenesis. In addition, CEACAM-1 has been reported to promote the apoptosis of various cells, including pulmonary and mammary epithelial cells, oral keratinocytes, cancer cells, Jurkat T cells, and cardiomyocytes $(13,15,16)$.

To date, the expression of CEACAM-1 in CRC has not been investigated. In the present study, CEACAM-1 expression was silenced in a CRC cell line, and its effects on cell growth and apoptosis were examined. The findings provide the first evidence that decreased CEACAM-1 expression promotes CRC progression.

\section{Materials and methods}

Cell culture. HCT-8 cells (Type Culture Collection of the Chinese Academy of Sciences, Shanghai, China) were maintained $\left(37^{\circ} \mathrm{C}, 5 \% \mathrm{CO}_{2}\right)$ in Dulbecco's modified Eagle's medium supplemented with $10 \%$ fetal calf serum (all from Gibco; Thermo Fisher Scientific, Inc., Waltham, MA, USA), $100 \mu \mathrm{g} / \mathrm{ml}$ penicillin, and $100 \mu \mathrm{g} / \mathrm{ml}$ streptomycin. The cells were passaged every 2-3 days, using 0.02\% EDTA and $0.1 \%$ trypsin.

Small-interfering RNA (siRNA) design and cell transfection. Vectors carrying either siRNA specific to CEACAM-1 or a negative control siRNA were generated by GeneChem Co., Ltd. (Shanghai, China). Three CEACAM-1-specific siRNAs were designed, as follows: siRNA1 sense, 5'-CAGCCACAGAAA UAAUUUATT-3' and antisense, 5'-UAAAUUAUUUCUGUG GCUGTT-3'; siRNA2 sense, 5'-CCGUCAAAUUGUAGGAUA UTT-3' and antisense, 5'-AUAUCCUACAAUUUGACGGTT-3'; and siRNA3 sense, 5'-GAGCUCUUUAUCCCUAACATT-3' and antisense, 5'-UGUUAGGGAUAAAGAGCUCTT-3'. A siRNA encoding a nonsense sequence (sense, 5'-UUCUCC GAACGUGUCACGUTT-3' and antisense, 5'-ACGUGACAC 
GUUCGGAGAATT-3'), was designed and used as a negative control (GenePharma Co., Ltd., Shanghai, China). HCT-8 cells were cultured until they reached $50 \%$ confluence, washed with PBS, and transfected with $50 \mathrm{nM}$ CEACAM-1-specific or control siRNA, according to the manufacturer's instructions. Cells were collected $48 \mathrm{~h}$ post-transfection, and maintained in fresh medium for $24 \mathrm{~h}$ prior to further experimentation and/or analysis.

Reverse transcription-quantitative polymerase chain reaction ( $R T-q P C R)$. CEACAM-1 expression was analyzed via RT-qPCR, as previously described (17). Briefly, total RNA was extracted from CRC cells, using an RNeasy Mini kit (Invitrogen; Thermo Fisher Scientific, Inc.). Genomic DNA was removed from the extracted total RNA via DNase I digestion, and then the total RNA was reverse transcribed to generate cDNA, according to the manufacturer's instructions (PrimeScript ${ }^{\mathrm{TM}}$ 1st strand cDNA Synthesis kit; Takara Biotechnology Co., Ltd., Dalian, China). qPCR was performed in triplicate in a $10 \mu 1$ reaction mix consisting of $4 \mu 1$ template DNA $(0.05 \mu \mathrm{g} / \mu \mathrm{l}), 5 \mu \mathrm{l} \mathrm{SYBR}-G r e e n$ (Takara Biotechnology Co., Ltd.), $0.2 \mu 1$ each forward and reverse oligonucleotide $(10 \mu \mathrm{M}$ each) and $0.6 \mu \mathrm{l}$ deionized water. The thermocycling conditions were as follows: $95^{\circ} \mathrm{C}$ for $10 \mathrm{~min}$, followed by 40 cycles of $95^{\circ} \mathrm{C}$ for $10 \mathrm{sec}$, $60^{\circ} \mathrm{C}$ for $30 \mathrm{sec}$, and $72^{\circ} \mathrm{C}$ for $30 \mathrm{sec}$. The primers used for qPCR were: CEACAM-1 forward, 5'-CAGGGGCTTCTG CTCACAGC-3' and reverse, 5'-AGTTGCTTCTTCACA AGAT-3'; $\beta$-actin forward, 5'-GGCTGTGGAGACAAA AATGACCTC-3' and reverse, 5'-AGGCTTGGGCTTGAA TGGAGTC-3'. The expression level was estimated with the $2^{-\Delta \Delta \mathrm{Cq}}$ method (18).

Western blot analysis. CEACAM-1 expression levels were determined via western blot analysis. CRC cells were washed twice with PBS, and collected via centrifugation. Proteins were then extracted with cell lysis buffer (CST Biological Reagents Company, Ltd., Shanghai, China) containing $1 \mathrm{mM}$ phenylmethylsulfonyl fluoride, and the protein concentration of each sample was determined using the BCA protein assay reagent kit (Beyotime Institute of Biotechnology, Lianyungang, China), and bovine serum albumin was used as a standard. The samples were denatured, and 50-80 $\mu \mathrm{g}$ were separated via polyacrylamide gel electrophoresis and transferred onto nitrocellulose membranes. The membranes were incubated first $\left(4^{\circ} \mathrm{C}\right.$, overnight) with anti-CEACAM-1 (cat. no. AF1857; Novus Biologicals,Ltd.,Cambridge,UK; dilution, 1:200) and anti- $\beta$-actin (cat. no. ab8227; Abcam, Cambridge, UK; dilution, 1:4,000) antibodies. The membranes were then incubated with horseradish peroxidase-conjugated rabbit anti-human secondary antibodies (cat. no. ab6759; Abcam; dilution, 1:3,000) for $12 \mathrm{~h}$ at $4^{\circ} \mathrm{C}$, and finally with enhanced chemiluminescence substrate (Bio-Rad Laboratories, Inc., Hercules, CA, USA). The resulting blots were analyzed using ImageJ software (NIH, Bethesda, MA, USA).

Cell proliferation. Cell proliferation was assessed by a CCK-8 assay (Roche Diagnostics GmbH, Mannheim, Germany), according to the manufacturer's instructions. Typically, cells were plated at a density of $1.5-2 \times 10^{3}$ cells/well
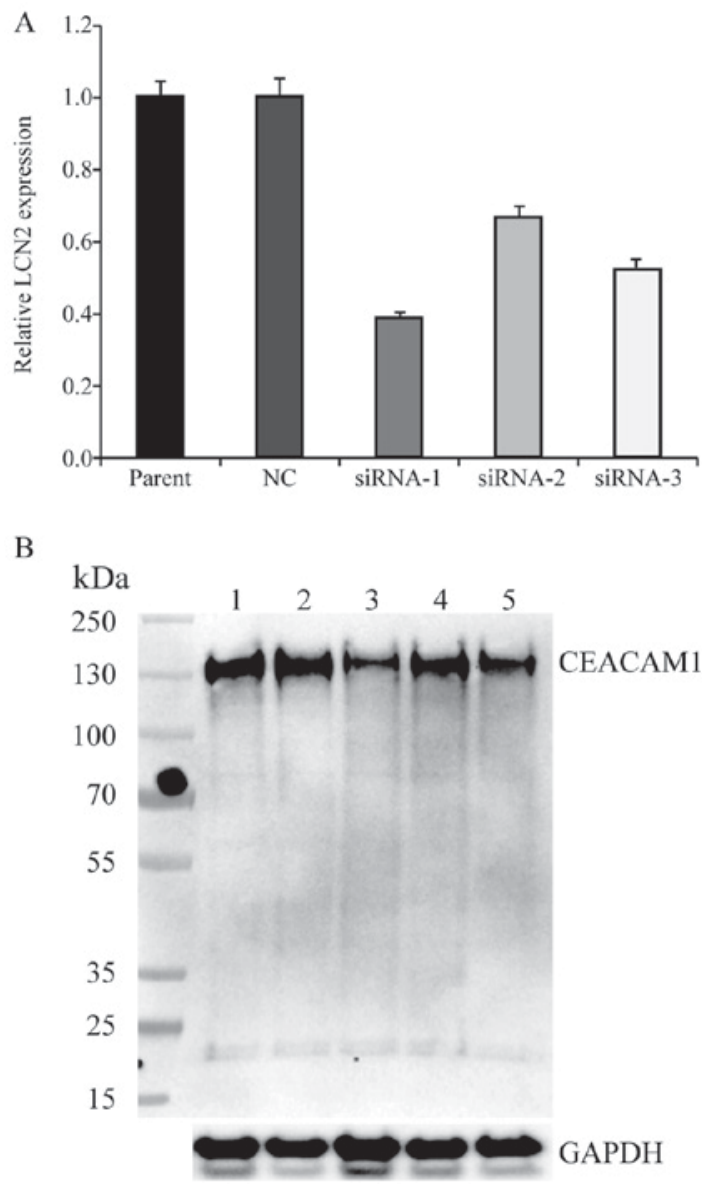

Figure 1. Knockdown of CEACAM-1 expression in HCT-8 cells (A) CEACAM-1 mRNA expression was suppressed using three different siRNA constructs in HCT-8 cells. Parental untreated cells and cells transfected with negative control (NC) siRNA were used as controls. The experiment was performed in triplicate. (B) Representative immunoblot of CEACAM-1 protein expression in HCT-8 cells, where GAPDH was used as a loading control. Lane 1, untreated group; lane 2, NC siRNA; lane 3, siRNA-1; lane 4, siRNA-2; lane 5, siRNA-3. CEACAM-1, carcinoembryonic antigen-related cell adhesion molecule 1; si, small interfering; NC, negative control.

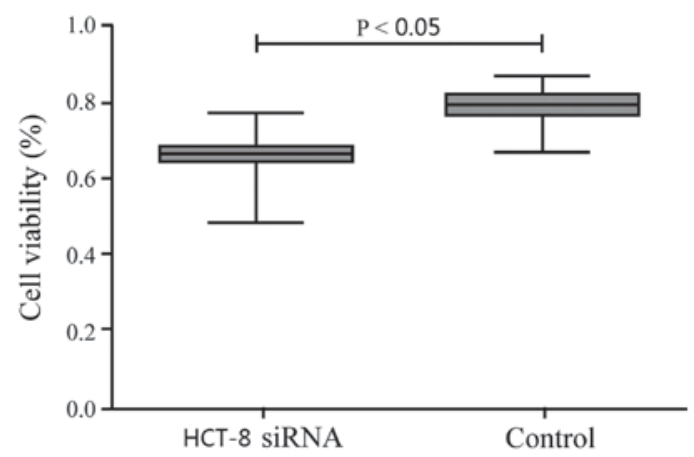

Figure 2. Downregulation of CEACAM-1 inhibits colorectal cancer cell proliferation. HCT-8 cells transfected with either CEACAM-1-siRNA or a negative control siRNA were assessed for cell proliferation using the CCK-8 assay. Results are presented relative to the control group. CEACAM-1, carcinoembryonic antigen-related cell adhesion molecule 1; si, small interfering.

in 96-well plates. After $48 \mathrm{~h}$ (to allow cell adherence to occur), cells were incubated with colorimetric substrates. Colorimetric changes were then measured in a multi-well 

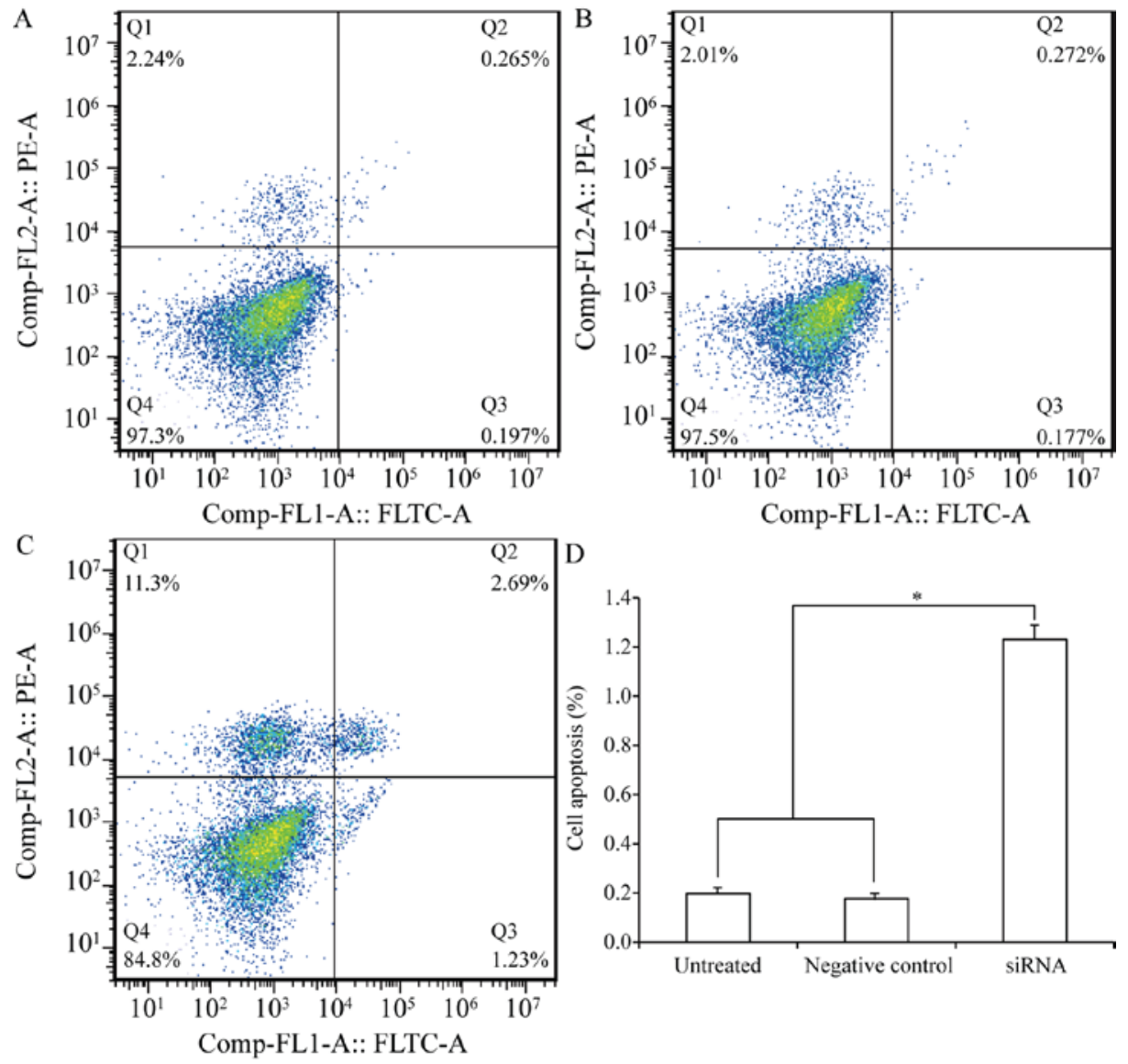

Figure 3. Downregulation of CEACAM-1 promotes colorectal cancer cell apoptosis. Representative plots are shown from flow cytometry analysis following Annexin V staining. (A) Untreated group. (B) Negative control siRNA group. (C) CEACAM-1-specific siRNA group. (D) Quantification of the results. "P<0.01. CEACAM-1, carcinoembryonic antigen-related cell adhesion molecule 1; si, small interfering.

spectrophotometer (MR5000 Multiplate Reader; Dynatech, Denkendorf, Germany), and cell survival following treatment was expressed as a \% of viable cells relative to control cell values. All experiments were independently conducted three times, and the results of the three experiments were then averaged.

Cell cycle assay. Cells were fixed, washed with PBS, treated with RNaseA, and stained $\left(37^{\circ} \mathrm{C}, 30 \mathrm{~min}\right)$ with $25 \mu \mathrm{g} / \mathrm{ml}$ propidium iodide (PI). The samples were then analyzed via flow cytometry, and the cell cycle phase distribution was quantified using Modfit Software (BD Biosciences, Franklin Lakes, NJ, USA). The proliferative index was calculated to represent the \% of cells identified to occur in the $S / G_{2} / M$ phase.

Apoptosis assay. Cells were collected, stained with Annexin V-fluorescein isothiocyanate (FITC) and 7-Aminoactinomycin D using an Annexin V-FITC Apoptosis Detection kit (KeyGen Biotech Co., Ltd., Nanjing, China) according to the manufacturer's instructions, and analyzed via flow cytometry (BD Biosciences).

Statistical analysis. All results are presented as the mean \pm standard error of the mean. Differences between two groups were evaluated by the unpaired Student's t-test. One-way analysis of variance with post-hoc analysis by
Bonferroni's test was performed to evaluate the data generated by the cellular viability and apoptosis assays. Additional statistical analyses were performed by Student's t-tests. $\mathrm{P}<0.05$ was considered to indicate a statistically significant difference.

\section{Results}

Knockdown of CEACAM-1 in HCT-8 cells. To investigate the function of CEACAM-1 expression in CRC cells, three separate siRNA sequences, comprising CEACAM-1-siRNA1, CEACAM-1-siRNA2, and CEACAM-1-siRNA3, were designed. When testing their efficacy, the three sequences induced a 61.3, 32.4 and $47.3 \%$ decrease in CEACAM-1 mRNA expression, respectively, in the CEACAM-1-knockdown cells compared with the negative control HCT-8 cells (Fig. 1A). The results from western blot analysis revealed a concordant reduction in CEACAM-1 protein expression levels compared with the control group (Fig. 1B). The CEACAM-1-siRNA1 sequence was selected for use in subsequent experiments, since it induced the greatest reduction in CEACAM-1 expression.

CEACAM-1 downregulation inhibits cell proliferation. CEACAM-1 knockdown significantly decreased the numbers of viable HCT-8 cells compared with the control group (Fig. 2), 

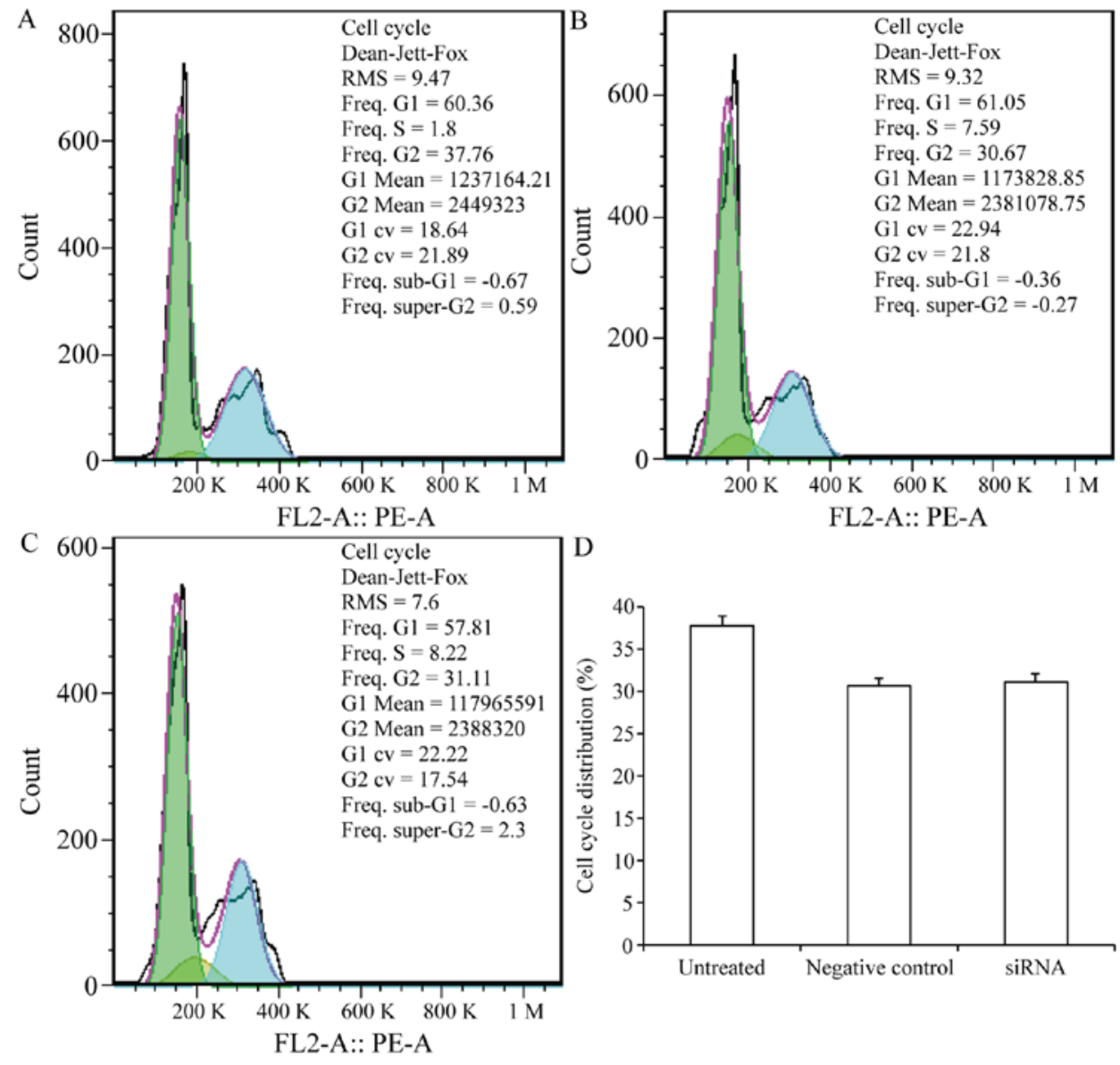

Figure 4. Effect of CEACAM-1 downregulation on the cell cycle distribution of colorectal cancer cells. Representative plots are shown from flow cytometry analysis following propidium iodide staining. (A) Untreated group.(B) Negative control siRNA group. (C) CEACAM-1-specific siRNA group.(D) Quantification of the results. CEACAM-1, carcinoembryonic antigen-related cell adhesion molecule 1; si, small interfering.

suggesting that CEACAM-1 silencing inhibited CRC cell proliferation.

CEACAM-1 downregulation increases cell apoptosis. Next, the effect of CEACAM-1 silencing on apoptosis was determined in CRC cells. The results from Annexin V cytometric analysis revealed that CEACAM-1 downregulation resulted in a significant increase in the apoptotic rate of HCT- 8 cells (1.23\% in the CEACAM-1-knockdown group compared with $0.197 \%$ in the control group; $\mathrm{P}<0.01$; Fig. 3). The experiment was performed in triplicate.

CEACAM-1 might function to inhibit carcinogenesis, which is often associated with both cell cycle arrest and activation of the cell death pathway. Therefore, the cell cycle phase distribution was examined in the CEACAM-1-knockdown cells to determine whether CEACAM-1 knockdown has an effect in cell cycle arrest. The results revealed no significant difference in the $\%$ of $\mathrm{G}_{2} / \mathrm{M}$-phase cells that occurred in the CEACAM-1-knockdown compared with the control group ( $\mathrm{P}>0.05$; Fig. 4). The experiment was performed in triplicate.

\section{Discussion}

To date, the function of CEACAM-1 expression in malignant tumors remains unclear. Previous studies have investigated CEACAM expression via either immunohistochemical or serum expression analyses $(19,20)$. To elucidate its functional role in $\mathrm{CRC}$, the present study assessed the effect of silencing CEACAM-1 expression on the viability and proliferation of a CRC cell line CEACAM-1 belongs to a diverse family of GPI-linked Igs that combine the structural features of the Ig superfamily with the functional properties of cadherins (21).

In the present study, CEACAM-1 expression was inhibited in the HCT- 8 cell line via three custom-designed siRNA sequences, achieving a maximal reduction in CEACAM-1 mRNA expression by $61.3 \%$ compared with the control cells, and a similar reduction in CEACAM-1 protein production. Subsequently, it was determined that CEACAM-1 downregulation significantly inhibited cell proliferation and promoted cell apoptosis, suggesting that CEACAM-1 may have a potential clinical use in the treatment of CRC. Consistent with these results, a previous study has demonstrated that CEACAM-1 knockdown results in the decreased proliferation and migration of human pancreatic adenocarcinoma Pac 5061 cells (17). Thus, it can be concluded that CEACAM-1 is likely an important modulator of CRC.

Collectively, the results of the present study demonstrated that CEACAM-1 downregulation in CRC cells significantly inhibited cell proliferation and promoted apoptosis. Thus, CEACAM-1 may be a critical mediator of CRC cell growth and progression, and as a result, a promising potential target for novel CRC treatment strategies. 


\section{Acknowledgements}

Not applicable.

\section{Funding}

The present study was supported by the Key Project of Universities and Colleges in Henan, China (grant no. $15 \mathrm{~A} 310033)$.

\section{Availability of data and materials}

The analyzed datasets generated during the study are available from the corresponding author on reasonable request.

\section{Authors' contributions}

Z-MH designed, analyzed the experiments and wrote the manuscript. H-MH and Y-WS performed the experiments and co-wrote the manuscript.

\section{Ethics approval and consent to participate}

Not applicable.

\section{Consent for publication}

Not applicable.

\section{Competing interests}

The authors declare that they have no competing interests.

\section{References}

1. Chen W, Zheng R, Zeng H and Zhang S: The incidence and mortality of major cancers in China, 2012. Chin J Cancer 35: 73 , 2016.

2. Rosso T, Malvezzi M, Bosetti C, Bertuccio P, Negri E and La Vecchia C: Cancer mortality in Europe, 1970-2009: An age, period, and cohort analysis. Eur J Cancer Prev 27: 88-102, 2016.

3. Cavallaro U and Christofori G: Cell adhesion in tumor invasion and metastasis: Loss of the glue is not enough. Biochim Biophys Acta 1552: 39-45, 2001.

4. Pignatelli M and Vessey CJ: Adhesion molecules: Novel molecular tools in tumor pathology. Hum Pathol 25: 849-856, 1994.

5. Oettle H, Post S, Neuhaus P, Gellert K, Langrehr J, Ridwelski K, Schramm H, Fahlke J, Zuelke C, Burkart C, et al: Adjuvant chemotherapy with gemcitabine vs observation in patients undergoing curative-intent resection of pancreatic cancer: A randomized controlled trial. JAMA 297: 267-277, 2007.

6. Rojas M, Fuks A and Stanners CP: Biliary glycoprotein, a member of the immunoglobulin supergene family, functions in vitro as a $\mathrm{Ca} 2(+)$-dependent intercellular adhesion molecule. Cell Growth Differ 1: 527-533, 1990.

7. Beauchemin N, Draber P, Dveksler G, Gold P, Gray-Owen S, Grunert F, Hammarström S, Holmes KV, Karlsson A, Kuroki M, et al: Redefined nomenclature for members of the carcinoembryonic antigen family. Exp Cell Res 252: 243-249, 1999.
8. Arabzadeh A, Chan C, Nouvion AL, Breton V, Benlolo S, DeMarte L, Turbide C, Brodt P, Ferri L and Beauchemin N: Host-related carcinoembryonic antigen cell adhesion molecule 1 promotes metastasis of colorectal cancer. Oncogene 32: 849-860, 2013.

9. Thöm I, Schult-Kronefeld O, Burkholder I, Schuch G, Andritzky B, Kastendieck H, Edler L, Wagener C, Bokemeyer C, Schumacher U and Laack E: Expression of CEACAM-1 in pulmonary adenocarcinomas and their metastases. Anticancer Res 29: 249-254, 2009.

10. Thies A, Moll I, Berger J, Wagener C, Brümmer J, Schulze HJ, Brunner $G$ and Schumacher U: CEACAM1 expression in cutaneous malignant melanoma predicts the development of metastatic disease. J Clin Oncol 20: 2530-2536, 2002.

11. Dango S, Sienel W, Schreiber M, Stremmel C, Kirschbaum A, Pantel K and Passlick B: Elevated expression of carcinoembryonic antigen-related cell adhesion molecule 1 (CEACAM-1) is associated with increased angiogenic potential in non-small-cell lung cancer. Lung Cancer 60: 426-433, 2008

12. Yoshikawa M, Morine Y, Ikemoto T, Imura S, Higashijima J, Iwahashi S, Saito YU, Takasu C, Yamada S, Ishikawa D, et al: Elevated preoperative serum CEA level is associated with poor prognosis in patients with hepatocellular carcinoma through the epithelial-mesenchymal transition. Anticancer Res 37: 1169-1175, 2017.

13. Li N, Yang JY, Wang XY, Wang HT, Guan BX and Zhou CJ: Carcinoembryonic antigen-related cell adhesion molecule 1 is expressed and as a function histotype in ovarian tumors. Ann Diagn Pathol 20: 7-12, 2016.

14. Gebauer F, Wicklein D, Horst J, Sundermann P, Maar H, Streichert T, Tachezy M, Izbicki JR, Bockhorn $M$ and Schumacher U: Carcinoembryonic antigen-related cell adhesion molecules (CEACAM) 1, 5 and 6 as biomarkers in pancreatic cancer. PLoS One 9: e113023, 2014.

15. Li Y and Shively JE: CEACAM1 regulates Fas-mediated apoptosis in Jurkat T-cells via its interaction with $\beta$-catenin. Exp Cell Res 319: 1061-1072, 2013.

16. Liu GX, Xie Q, Zhou CJ, Zhang XY, Ma BL, Wang CQ, Wei FC, Qu X and Sun SZ: The possible roles of OPN-regulated CEACAM1 expression in promoting the survival of activated $\mathrm{T}$ cells and the apoptosis of oral keratinocytes in oral lichen planus patients. J Clin Immunol 31: 827-839, 2011.

17. Beauchemin $\mathrm{N}$ and Arabzadeh A: Carcinoembryonic antigen-related cell adhesion molecules (CEACAMs) in cancer progression and metastasis. Cancer Metastasis Rev 32: 643-671, 2013.

18. Livak KJ and Schmittgen TD: Analysis of relative gene expression data using real-time quantitative PCR and the 2(-Delta Delta C(T)) method. Methods 25: 402-408, 2001.

19. Simeone DM, Ji B, Banerjee M, Arumugam T, Li D, Anderson MA, Bamberger AM, Greenson J, Brand RE, Ramachandran V and Logsdon CD: CEACAM1, a novel serum biomarker for pancreatic cancer. Pancreas 34: 436-443, 2007.

20. Blumenthal RD, Leon E, Hansen HJ and Goldenberg DM: Expression patterns of CEACAM5 and CEACAM6 in primary and metastatic cancers. BMC Cancer 7: 2, 2007.

21. Hauck W, Nédellec P, Turbide C, Stanners CP, Barnett TR and Beauchemin N: Transcriptional control of the human biliary glycoprotein gene, a CEA gene family member down-regulated in colorectal carcinomas. Eur J Biochem 223: 529-541, 1994.

(i) () $९$ This work is licensed under a Creative Commons Attribution-NonCommercial-NoDerivatives 4.0 International (CC BY-NC-ND 4.0) License. 